Review began 08/25/2021 Review ended 09/12/2021 Published 09/19/2021

(c) Copyright 2021

Kelkar et al. This is an open access article distributed under the terms of the Creative Commons Attribution License CC-BY 4.0. which permits unrestricted use, distribution, and reproduction in any medium, provided the original author and source are credited.

\section{Association of Tumor Lysis Syndrome and Metastatic Melanoma}

\author{
Neil Kelkar $^{1}$, Jue Wang ${ }^{2,3}$ \\ 1. Oncology, University of Arizona College of Medicine - Phoenix, Phoneix, USA 2. Genitourinary Oncology, Dignity \\ Health Cancer Institute, St. Joseph's Hospital and Medical Center, Phoenix, USA 3. Genitourinary Oncology, Creighton
} University School of Medicine, St. Joseph's Hospital and Medical Center, Phoenix, USA

Corresponding author: Neil Kelkar, nkelkar@email.arizona.edu

\section{Abstract \\ Background}

Tumor lysis syndrome (TLS) is a known oncologic emergency characterized by severe metabolic derangements. TLS has been well documented in patients with hematologic malignancies, but rarely with metastatic melanoma. The objective of this study was to investigate the clinical characteristics and outcomes of TLS with metastatic melanoma.

\section{Methods}

Retrospective literature review and analysis.

\section{Results}

Eighteen cases of TLS were identified with metastatic melanoma from published literature. The median age of patients was 63 years (36-79 years). All patients have stage IV disease. Seven cases (39\%) of TLS were associated with multiple treatment regimes, including nivolumab (22\%), ipilimumab (16\%), and dacarbazine (22\%). The time from treatment to diagnosis was 3.5 days (8 hours-21 days) in treatment-related TLS. Three cases (17\%) were due to spontaneous TLS. The majority of cases have a high tumor burden (77.5\%) and liver metastasis (83\%). Seven cases were treated with rasburicase (39\%). The mortality rate was $100 \%$ for the patients with spontaneous TLS and 73\% for patients with treatment-related TLS. Three cases utilized traditional chemotherapy and the six most recent cases of treatment-associated TLS utilized immunotherapy and targeted therapy.

\section{Conclusion}

TLS in metastatic melanoma, due to either spontaneous or treatment-related causes, is associated with a very high mortality rate. This study highlights the importance of awareness, early intervention, and risk assessment of this underdiagnosed emergency.

Categories: Oncology

Keywords: tumor-lysis syndrome, malignant melanoma metastasis, tumor lysis in solid tumors, mortality in melanoma, immuno-checkpoint inhibitor

\section{Introduction}

Tumor lysis syndrome (TLS) is a serious, life-threatening oncological emergency due to the massive cellular lysis and release of contents into the bloodstream. This results in severe metabolic derangement including hyperkalemia, hyperphosphatemia, hyperuricemia, and hypocalcemia [1]. Although TLS is a wellcharacterized complication for hematologic malignancies such as acute lymphoblastic leukemia, it is underreported in solid tumors [2]. Between 1994 and 2020, we identified 18 different cases of TLS associated with malignant melanoma [3-18]. The objective of this study was to examine published information on management, characteristics, and outcomes of patients with TLS associated with malignant melanoma.

\section{Materials And Methods}

\section{Literature search}

This is a retrospective systematic review of published cases of patients with metastatic cancer who were diagnosed with TLS. This was performed by searching the database PubMed for articles published from January 1991 to May 2021. Search terms included "tumor lysis syndrome," "solid tumor," and "melanoma." The resulting case reports were reviewed, and additional articles of interest were identified from reference lists. 


\section{Cureus}

\section{Data collection and analysis}

Information regarding the patients including gender, age at diagnosis, presentation, associated medical history (such as prior renal function), tumor characteristics (symptoms, pathology, stage), treatment, laboratory results, and outcomes (response, adverse effects) were recorded when available. Statistics such as medians, ranges, and frequency counts were used to assess the pooled sample.

\section{Results}

A total of 18 cases of metastatic melanoma-related TLS (15 cases treatment-related and three cases of spontaneous TLS [sTLS]) were identified from 17 case reports. The demographic, symptoms, and survival outcomes are summarized in Table 1 .

\begin{tabular}{|c|c|c|c|c|c|c|}
\hline Author & Year & Age/Gender & Treatment & Liver Metastasis & Rasburicase & Outcome \\
\hline Minasian [3] & 1994 & 76/M & Tumor necrosis factor-a, anti-GD3 ganglioside MoAb & Yes & No & Death \\
\hline Castro [4] & 1999 & $61 / M$ & IL-2, interferon-a, cisplatin, vinblastine, and dacarbazine & Yes & No & Death \\
\hline Stoves [5] & 2001 & $41 / \mathrm{M}$ & Cisplatin, interferon-a, dacarbazine & Yes & No & Death \\
\hline Habib [6] & 2002 & $56 / \mathrm{F}$ & Hydrocortisone & No & No & Survival \\
\hline Busam [7] & 2004 & $36 / F$ & Cisplatin, vinblastine, dacarbazine, interferon alpha & Yes & No & Survival \\
\hline Borne $[9]$ & 2009 & $42 / M$ & High-dose sterolds & Yes & Yes & Death \\
\hline Song [10] & 2011 & 46/M & Spontaneous & Yes & No & Death \\
\hline Mouallem [11] & 2013 & 69/M & Spontaneous & Yes & No & Death \\
\hline Mouallem [11] & 2013 & $68 / \mathrm{M}$ & Prednisone and dacarbazine & Yes & No & Death \\
\hline $\operatorname{Dar}[12]$ & 2014 & $65 / M$ & Palliative radiation therapy & Yes & Yes & Death \\
\hline Meeks [18] & 2016 & 46/M & Dexamethasone & Yes & Yes & Death \\
\hline Masson [8] & 2017 & $71 / \mathrm{M}$ & Ipilimumab & Yes & Not recorded & Death \\
\hline Brunnhoelz [14] & 2018 & $76 / \mathrm{M}$ & Nivolumab & Yes & Yes & Death \\
\hline Valle [15] & 2019 & 65/M & Spontaneous & Yes & Yes & Death \\
\hline Sugimoto [16] & 2020 & 79/M & Nivolumab & Yes & No & Death \\
\hline Magara [17] & 2020 & $45 / M$ & Nivolumab + iplilmumab & No & Yes & Survival \\
\hline Byron [13] & 2020 & $48 / \mathrm{F}$ & Encorafenib + binimetinib & No & Yes & Survival \\
\hline Konishi [19] & 2020 & 69/M & Nivolumab + ipilimumab & Yes & Yes & Death \\
\hline
\end{tabular}

\section{TABLE 1: Overview of published cases of tumor lysis syndrome in patients with metastatic} melanoma

Patients in the study had a median age of 63 years (36-79) and showed a male predominance (77.6\%). Majority of patients presented with liver metastasis (83\%) and high tumor burden (77.5\%). Patients were noted to have elevated lactate dehydrogenase (LDH) with other biochemical indications of TLS such as uric acid, potassium, and phosphorus. Fifteen cases (83\%) of TLS were associated with a variety of treatment options including nivolumab (22\%), ipilimumab (11\%), dacarbazine (22\%), cisplatin (17\%), vinblastine (11\%), and interferon-a (11\%).

The median time from treatment to diagnosis of TLS was 3.5 days ( 8 hours-21 days). Three cases (17\%) were due to sTLS. Seven cases were treated with rasburicase (39\%). The mortality rate was $73 \%$ for patients with treatment-related TLS and $100 \%$ for patients with sTLS.

\section{Discussion}

TLS is a well-known emergency in hematological malignancies. TLS was considered rare in solid tumors, as effective pharmacological treatments were not available. However, rates have been significantly increasing as therapies become more effective [2]. TLS can hypothetically occur in every tumor type, as the tumor 
burden is considered a more important factor than the origin of the tumor tissue (18). High tumor burden is defined as having three specific measurements: elevated LDH ( $>2 \mathrm{x}$ the upper limit of normal), bulky disease $(>10 \mathrm{~cm})$, and elevated leukocytes $(>25,000)$. Studies have also characterized tumor burden by the evidence of distant metastases [20,21]. Our study found that all studies, which reported an LDH reading, had a reading above two times the upper normal limit.

Melanoma is the least common, but the most malignant lesion of the skin. The mortality rate of melanoma differs with age and gender, but recent studies place the rate around 2.7/100,000 cases [22]. However, TLS due to melanoma is frequently misdiagnosed. TLS is often misclassified as acute kidney injury or other causes of electrolyte abnormalities. Additionally, adverse effects of pharmaceuticals during clinical trials are frequently underreported [23]. We believe that the incidence of TLS due to melanoma is underreported and underdiagnosed, and, therefore, prevents clinicians from understanding the true epidemiology of this emergency [3-17].

The study outlines several notable findings. First, TLS in metastatic melanoma carries a worse prognosis when compared to hematological malignancies. TLS morality for hematological cancer is approximately $21 \%$ for hematological cancers, but our study shows a mortality rate of $73 \%$ for treatment-induced TLS and $100 \%$ for sTLS [24]. This is likely due to a lack of awareness of TLS in metastatic melanoma, and other solid tumors. The result is a delayed diagnosis and inadequate management, leading to a higher mortality rate for this population. Second, TLS commonly occurs in patients who have advanced cancer with large disease burdens. Liver metastases were documented in 17 patients (Table 1), showing an increased risk of developing TLS [25]. The most important finding from examining studies is that TLS due to metastatic melanoma occurs at a median of 3.5 days with a range of 8 hours to 21 days. This suggests that TLS rapidly develops due to effective therapies. For patients who are at increased risk of developing TLS, we advise monitoring patients' electrolytes and renal function during the first few weeks of therapy.

Current guidelines and management of TLS, which are based on pediatric and adult hematological malignancies, were established 13 years ago, and at that time, effective therapies for metastatic melanoma and other solid tumors were not available. Our study found that most TLS associated with metastatic melanoma were detected recently when effective treatment was first available. Only three studies utilized traditional chemotherapy. However, 16/18 (89\%) cases were reported after the year 2000, and the six most recent cases of treatment-associated TLS utilize immunotherapy and targeted therapy. This suggests that improved treatment modalities have increased rates in solid organ tumors. The mechanism of checkpoint inhibitors, such as ipilimumab, has not fully been revealed. It is believed that checkpoint inhibitors lead to T-cell activation and cause cytokine-dependent endothelial toxicity and massive destruction of tumor cells $[14,26]$.

\section{Conclusions}

This review highlights the nature of TLS, an under-recognized emergency due to metastatic melanoma. It is essential that clinicians recognize that TLS may occur shortly after treatment and spontaneously. There is a need to redefine the risk factors and guidelines of TLS in the era of targeted and immunotherapy.

\section{Additional Information \\ Disclosures}

Human subjects: Consent was obtained or waived by all participants in this study. Animal subjects: All authors have confirmed that this study did not involve animal subjects or tissue. Conflicts of interest: In compliance with the ICMJE uniform disclosure form, all authors declare the following: Payment/services info: All authors have declared that no financial support was received from any organization for the submitted work. Financial relationships: All authors have declared that they have no financial relationships at present or within the previous three years with any organizations that might have an interest in the submitted work. Other relationships: All authors have declared that there are no other relationships or activities that could appear to have influenced the submitted work.

\section{References}

1. Howard SC, Pui C-H, Ribeiro RC: Chapter 4 - Tumor lysis syndrome. Renal Disease in Cancer Patients [Internet]. Finkel KW, Howard SC (ed): Academic Press, Cambridge, MA; 2014. 39-64.

2. McBride A, Westervelt $P$ : Recognizing and managing the expanded risk of tumor lysis syndrome in hematologic and solid malignancies. J Hematol Oncol. 2012, 5:75. 10.1186/1756-8722-5-75

3. Minasian LM, Szatrowski TP, Rosenblum M, et al.: Hemorrhagic tumor necrosis during a pilot trial of tumor necrosis factor-alpha and anti-GD3 ganglioside monoclonal antibody in patients with metastatic melanoma. Blood. 1994, 83:56-64.

4. Castro MP, VanAuken J, Spencer-Cisek P, Legha S, Sponzo RW: Acute tumor lysis syndrome associated with concurrent biochemotherapy of metastatic melanoma: a case report and review of the literature. Cancer. 1999, 85:1055-9.

5. Stoves J, Richardson D, Patel H: Tumour lysis syndrome in a patient with metastatic melanoma treated with biochemotherapy. Nephrol Dial Transplant. 2001, 16:188-9. 10.1093/ndt/16.1.188 
6. Habib GS, Saliba WR: Tumor lysis syndrome after hydrocortisone treatment in metastatic melanoma: a case report and review of the literature. Am J Med Sci. 2002, 323:155-7. 10.1097/00000441-200203000-00007

7. Busam KJ, Wolchok J, Jungbluth AA, Chapman P: Diffuse melanosis after chemotherapy-induced tumor lysis syndrome in a patient with metastatic melanoma. J Cutan Pathol. 2004, 31:274-80. 10.1111/j.03036987.2003.00154.x

8. Masson Regnault M, Ofaiche J, Boulinguez S, et al.: Tumour lysis syndrome: an unexpected adverse event associated with ipilimumab. J Eur Acad Dermatol Venereol. 2017, 31:e73-4. 10.1111/jdv.13733

9. Borne E, Desmedt E, Duhamel A, et al.: Oral metronomic cyclophosphamide in elderly with metastatic melanoma. Invest New Drugs. 2010, 28:684-9. 10.1007/s10637-009-9298-5

10. Song M, Chan CC, Stoeckel DA: Spontaneous tumor lysis syndrome in metastatic melanoma . World J Oncol. 2011, 2:204-7. 10.4021/wjon347w

11. Mouallem M, Zemer-Wassercug N, Kugler E, Sahar N, Shapira-Frommer R, Schiby G: Tumor lysis syndrome and malignant melanoma. Med Oncol. 2013, 30:364. 10.1007/s12032-012-0364-z

12. Dar L, Gendelman O, Amital H: Tumor lysis syndrome presenting in a patient with metastatic melanoma treated with radiation therapy. Isr Med Assoc J. 2014, 16:456-7.

13. Byron Y, Nott L, Shackleton M: Case report: acute tumour lysis syndrome following encorafenib and binimetinib for v600E metastatic melanoma with large intra-abdominal mass. Melanoma Res. 2020, 30:6257. 10.1097/CMR.0000000000000696

14. Brunnhoelzl D, Wang J: Acute tumor lysis syndrome after anti-pd-1 immunotherapy nivolumab for metastatic melanoma. J Mol Oncol Res. 2017, 1:

15. Valle KD, Issa M: When you can't blame the chemo: an emergent case of acute kidney injury . Minn Med. 2020 ,

16. Sugimoto S, Terashima T, Yamashita T, et al.: Tumor lysis syndrome in a patient with metastatic melanoma treated with nivolumab. Clin J Gastroenterol. 2020, 13:935-9. 10.1007/s12328-020-01164-x

17. Magara A, Kato H, Oda T, Nakamura M, Komatsu H, Morita A: Tumor lysis syndrome associated with nivolumab plus ipilimumab combination therapy in a melanoma patient. J Dermatol. 2020, 47:e411-2. 10.1111/1346-8138.15547

18. Meeks MW, Hammami MB, Robbins KJ, Cheng KL, Lionberger JM: Tumor lysis syndrome and metastatic melanoma. Med Oncol. 2016, 33:134. 10.1007/s12032-016-0854-5

19. Konishi R, Ishitsuka Y, Imai H, et al.: Tumor lysis syndrome and collateral immune activation in dual checkpoint blockade. J Cutan Immunol Allergy. 2021, 4:39-40. 10.1002/cia2.12148

20. Wang JF, Luther RD, Mass D, Wang J: Tumor lysis syndrome associated with renal cell carcinoma . Int Med. 2019, 1:97-100.

21. Coiffier B, Altman A, Pui CH, Younes A, Cairo MS: Guidelines for the management of pediatric and adult tumor lysis syndrome: an evidence-based review. J Clin Oncol. 2008, 26:2767-78. 10.1200/JCO.2007.15.0177

22. Guy GP Jr, Thomas CC, Thompson T, Watson M, Massetti GM, Richardson LC: Vital signs: melanoma incidence and mortality trends and projections - United States, 1982-2030. MMWR Morb Mortal Wkly Rep. 2015, 64:591-6.

23. Montané E, Santesmases J: Adverse drug reactions. Med Clin (Barc). 2020, 154:178-84. 10.1016/j.medcli.2019.08.007

24. Adeyinka A, Bashir K: Tumor lysis syndrome. StatPearls [Internet]. StatPearls Publishing, Treasure Island, FL; 2021.

25. Harmon J, Allen S, Cassaday J, et al.: Liver metastasis as an independent predictor for mortality in patients who developed tumor lysis syndrome: analysis of 132 patients with solid tumors. J Clin Oncol. 2018, 36:e18766.

26. Cordrey EO, Wang J: Tumor lysis syndrome associated with immune checkpoint blockade in solid tumors . Jpn J Cancer Oncol Res. 2018, 1:1005. 